\title{
In search for biomarkers and potential drug targets for uterine serous endometrial cancer
}

\author{
Giorgia Dinoi ${ }^{1,2} \cdot$ Andrea Mariani $^{2} \cdot$ Enrica Martinelli $i^{3,4} \cdot$ Alessandra Ciucci $^{3,4} \cdot$ Gian Franco Zannoni $^{5}$. \\ Amy L. Weaver ${ }^{6}$. Gary L. Keeney ${ }^{7} \cdot$ George Vasmatzis $^{8} \cdot$ Panos Z. Anastasiadis $^{9} \cdot$ Francesco Fanfani $^{1}$. \\ Giovanni Scambia ${ }^{1}$ (D) Daniela Gallo ${ }^{3,4}$
}

Received: 19 January 2021 / Accepted: 10 February 2021 / Published online: 23 March 2021

(c) The Author(s) 2021

\begin{abstract}
Objective Serous endometrial cancer (USC) is a challenging malignancy associated with metastasis, recurrence and poor outcome. To identify clinically relevant prognostic biomarkers, we focused on a panel of proteins selected after a comprehensive literature review, for tumour profiling of a homogeneous cohort of USC patients.

Methods Protein levels and localization were assessed by immunohistochemistry analysis in 36 hysterectomy samples. Tissue sections were stained with the following antibodies: Aurora A, phospho (T288) Aurora A, BRCA1, CHK1, CIP2A, Cyclin B1, Cyclin E, E2F-1, phospho (S364) E2F-1, FBXW7, FOXM1, phospho (S9) GSK3Beta, PLK1, phospho (T210) PLK1, PPP2R1B, p73, RAD51. Each marker was evaluated as a continuously-scaled variable for association with disease progression and death, using Cox proportional hazards models. The sample consisted of 36 patients with USC, half with stage III or IV disease.

Results Results showed that higher CHK1 (Checkpoint kinase 1) expression was associated with a decreased risk of progression and death, after adjusting for stage. Interestingly, analysis of a TCGA data set of 109 USC patients corroborates our results showing a favourable prognostic role of CHEK1 after adjusting for stage. Higher FBXW7 (F-box and WD repeat domain containing 7) expression and higher cytoplasmic expression of PPP2R1B (Protein Phosphatase 2 A, Scaffold Subunit Abeta) were each associated with a decreased risk of progression, after adjusting for stage.

Conclusions In conclusion, results from the present study identify new clinically relevant biomarkers and potential drug targets for uterine serous endometrial cancer.
\end{abstract}

Keywords Serous endometrial cancer $\cdot$ Uterine malignancy $\cdot$ Biomarkers $\cdot$ Immunochemistry $\cdot$ Molecular profiling $\cdot$ Drug targets

Giovanni Scambia

giovanni.scambia@policlinicogemelli.it

1 Gynecology Oncology Unit, Department of Woman, Child and Public Health, Fondazione Policlinico Universitario A. Gemelli IRCCS, Largo A. Gemelli, 8, 00168, Rome, Italy

2 Division of Gynaecologic Surgery, Department of Obstetrics and Gynaecology, Mayo Clinic, Rochester, MN, USA

3 Department of Life Sciences and Public Health, Section of Gynaecology and Obstetrics, Università Cattolica del Sacro Cuore, Rome, Italy

4 Unit of Translational Medicine for Woman and Child Health, Department of Woman, Child and Public Health, Fondazione Policlinico Universitario A. Gemelli IRCCS, Rome, Italy
5 Gynecopathology and Breast Pathology Unit, Department of Woman, Child and Public Health, Fondazione Policlinico Universitario A. Gemelli IRCCS, Rome, Italy

6 Division of Clinical Trials and Biostatistics, Department of Quantitative Health Sciences, Mayo Clinic, Rochester, MN, USA

7 Division of Anatomic Pathology, Mayo Clinic Rochester, Rochester, MN, USA

8 Department of Molecular Medicine, Department of Laboratory Medicine \& Pathology, Mayo Clinic, Rochester, MN, USA

9 Department of Cancer Biology, Mayo Clinic, Jacksonville, FL, USA 


\section{Introduction}

Endometrial cancer (EC) is the most common gynaecological cancer in the developed world and the fourth most common cancer in women (Gentry-Maharaj and Karpinskyj 2020). Based on differences in histology and clinical outcomes, endometrial cancers have long been divided into the estrogen-dependent type I (endometrioid adenocarcinoma) with a favourable prognosis, and the estrogenindependent type II (predominantly serous and clear cell carcinoma) (Bokhman 1983; Dedes et al. 2011). Approximately $80-90 \%$ of EC are endometrioid-type carcinomas, and $2-10 \%$ are serous endometrial carcinomas (USC) (Dedes et al. 2011). Although these latter account for only a small percentage of EC, as high as $40 \%$ of EC deaths are attributed to USC (Hamilton et al. 2008; Del Carmen et al. 2012). This highly aggressive behaviour is mainly related to its tendency to metastasize, even when the primary tumour is small (Hamilton et al. 2006). Currently, the standard of care for most patients with USC includes surgery and platinum-based adjuvant chemotherapy with or without radiotherapy. Indeed, given its distinct biological behaviour, USC is typically excluded from large clinical trials (Keys et al. 2004; Nout et al. 2010).

During the last decade, we have significantly increased our understanding of biological pathways implicated in disease development and progression. Specifically, USC were found to harbour a specific genomic signature, which is different from other EC histologic subtypes, and it is partially overlapping with high-grade serous ovarian carcinoma (HGSOC) and basal-like breast cancer (Kuhn et al. 2012; Le Gallo et al. 2012; Cancer Genome Atlas Research Network 2013). Mutations in the TP53 (Tumor protein p53) tumour suppressor gene and/or stabilization of the p53 protein were the most frequent molecular aberrations in serous carcinomas, occurring at frequencies in excess of $85 \%$. Notably, however, the mutation frequency of five genes [PIK3CA (Phosphatidylinositol-4,5-Bisphosphate 3-Kinase Catalytic Subunit Alpha), PIK3R1 (phosphoinositide-3-kinase regulatory subunit 1), PTEN (phosphatase and tensin homolog), PPP2R1A (protein phosphatase 2 scaffold subunit Aalpha) and FBXW7 (F-box and WD repeat domain containing 7)] was dramatically higher in USC as compared to serous ovarian cancer (Cancer Genome Atlas Research Network 2013). These five genes are part of the PI3K (phosphatidylinositol 3-kinase)AKT (protein kinase B)-FBXW7 pathway. The FBXW7 tumour suppressor is a component of the FBXW7-SKP1 (S-phase kinase-associated protein 1)-CUL1 (Cullin-1) ubiquitin ligase complex, which mediates the ubiquitination of the protein products of multiple oncogenes, including cyclin E, AURKA (Aurora A kinase), PLK1 (Polo-like kinase-1) and FOXM1 (Forkhead box protein M1) among the others (Galindo-Moreno et al. 2020). Glycogen synthase kinase-3 (GSK3)-mediated phosphorylation of the targeted proteins is a necessary step for subsequent FBXW7-mediated ubiquitylation and proteasomal degradation (Hoxhaj and Manning 2020). GSK3 is a ubiquitously expressed protein kinase that exists in two isoforms, $\alpha$ and $\beta$. The protein is active under basal conditions and is inhibited in response to growth factors and insulin, via AKT-mediated phosphorylation. GSK3 and FBXW7 thus act in concert to regulate ubiquitination of many important factors associated with cell division and growth.

Pathway analysis and clustering of the endometrial tumours in the TCGA data set also revealed that dysregulation of mitotic processes is a frequent occurrence in USC (Cancer Genome Atlas Research Network 2013). In this context, it is worth noting that numerous studies have established a crucial role for E2F-1 (E2F transcription factor 1) in the control of cellular proliferation, through transcriptional activation of genes important for cell-cycle progression, such as cyclin B1 and cyclin E (Hallstrom et al. 2008). Therefore, E2F-1 may have profound implications in USC, acting as a master regulator of cell fate. In line with this concept, E2F-1 has been identified as a prognostic biomarker in endometrial cancer, being mostly expressed in the serous histotype (Alkushi et al. 2007). Actually, E2F-1 has been shown to induce both proliferation and apoptosis, being the balance of these events regulated by the PI3K/AKT signalling, that would selectively block the expression of genes in the apoptotic program, but not in the proliferative program (Hallstrom et al. 2008). TP73 (Tumor protein p73) is a target of E2F-1 apoptotic program and altered TP73 expression (due to aberrant DNA methylation) is considered a predictor of USC histology (Seeber et al. 2010). Notably, after DNA damage, $\mathrm{p} 73$ induction is regulated by the checkpoint kinases CHK1 and CHK2, through E2F-1 stabilization, in a pathway central to p53-independent apoptosis (Urist et al. 2004). Therefore, this signalling may be a major determinant of anti-cancer drug efficacy in USC patients carrying TP53 mutations. Overall, these data suggest that molecular pathways converging on p73 may play important roles in driving disease outcome.

TCGA data also demonstrated that up to $40 \%$ of type II EC tumours are associated with heterozygous missense mutations in PPP2R1A, an established tumour suppressor gene encoding the A $\alpha$ subunit of PP2A (Protein Phosphatase 2A), one of the major cellular serine-threonine phosphatases, involved in the regulation of PI3K/AKT pathway (Kuo et al. 2008; Remmerie and Janssens 2019). It has been recently shown that also PPP2R1B (the gene encoding for the $\beta$ isoform of subunit $A$ ) is mutated in human neoplasm, with a functional inactivation of the protein that may promote tumorigenesis, through its role in cell-cycle regulation 
and cellular growth control (Calin et al. 2000). The inclusion of both isoforms of subunit $\mathrm{A}$ in the genes mutated in human cancer support the potential role of PP2A in human tumorigenesis via a mechanism of inactivation of the phosphatase activity (Calin et al. 2000). In line with these findings, overexpression of CIP2A (Cellular Inhibitor of PP2A, also called p90), an endogenous PP2A-inhibitory protein, has been associated to worse outcome in gynaecologic cancers (Remmerie and Janssens 2019).

Finally, recent data supported the view that homologous recombination deficiency (HRD) occurs in endometrial cancers and is largely restricted to non-endometrioid, TP53-mutant endometrial cancers, the spectrum of germline mutations detected including BRCA1/2 (breast cancer type $1 / 2$ susceptibility protein) and RAD51 (DNA repair protein RAD51 homolog 1) among others (Ring et al. 2016; De Jonge et al. 2019).

Based on the above-mentioned, well-known or emerging evidence of molecular determinants of cancer development, we selected a panel of proteins for tumour profiling of a homogeneous cohort of USC patients, to identify clinically relevant prognostic biomarkers. Results obtained provide new perspective and potential strategies for drug target discovery in USC therapy.

\section{Materials and methods}

\section{Patients}

We analysed a retrospective series of patients with pathological confirmed diagnosis of serous endometrial cancer, who underwent surgery between January, 2009 and December, 2012 at Mayo Clinic (Rochester, Minnesota). Approval was provided by the Institutional Review Board (IRB) at Mayo Clinic. Clinical information was obtained from the existing medical records in accordance with the institutional guidelines. Data abstracted from the medical records included demographic (e.g., age at surgery, BMI) as well as surgical and pathological details [e.g., surgical approach, histology, International Federation of Gynecology and Obstetrics (FIGO) grade and stage, tumour diameter, lymph-vascular space invasion (LVSI), myometrial invasion, peritoneal cytology, pelvic and para-aortic lymph nodes status]. Patients who did not give research authorization, with synchronous cancers, or who underwent neoadjuvant chemotherapy were excluded. Formalin-fixed paraffin-embedded (FFPE) tissue blocks were cut as consecutive sections and slides sent to the Gynaecology Oncology Unit, Fondazione Policlinico A. Gemelli IRCCS, Rome, Italy, for immunohistochemical staining and analysis of the selected protein panel.

\section{Immunohistochemistry}

A board-certified gynaecologic pathologist (GFZ) reviewed all tumours to confirm the diagnosis of USC. Tissue sections were stained with the following antibodies: Aurora A, phospho (T288) Aurora A, BRCA1, CHK1, CIP2A/p90, Cyclin B1, Cyclin E, E2F-1, phospho (S364) E2F-1, FBXW7, FOXM1, phospho (S9) GSK3Beta, PLK1, phospho (T210) PLK1, PPP2R1B, p73 and RAD51.

The process of deparaffinization, rehydration and epitope retrieval of tissue specimens was performed with low or high pH Target Retrieval Solution (Agilent Technologies, Santa Clara, CA, USA) in DAKO PT Link module (Agilent Technologies). The endogenous peroxidase was blocked with $3 \%$ $\mathrm{H}_{2} \mathrm{O}_{2}$ for $5 \mathrm{~min}$. To reduce non-specific binding, sections were incubated with $20 \%$ normal goat serum for $30 \mathrm{~min}$, at room temperature, and then with primary antibody in a humidified chamber. Conditions for antigen retrieval, incubation times, and the primary antibodies used are described in Table S1 (see Supplement). Sections were incubated with the secondary antibody, anti-mouse/rabbit EnVision SystemHRP (Dako, Agilent) for $30 \mathrm{~min}$, at room temperature. The slides were developed with diaminobenzidine (DAB substrate System, Dako, Agilent), counterstained with Mayer's Haematoxylin, dehydrated in ethanol and xylene and mounted. Staining without primary antibody was used to validate the specificity of the secondary antiserum, while a section from a tissue known to express the protein of interest was used as positive control. Expression was evaluated by considering the percentage of cells exhibiting immunoreaction, as well as the localization of signalling (nuclear and/ or cytoplasmic). Graphical analysis was performed using GraphPad Prism 6 (GraphPad Software, Inc. La Jolla, CA, USA). Two independent observers (GFZ and EM), who were unaware of the patients' outcome, evaluated immunostained tissue sections.

\section{Statistical analysis of the study sample}

Baseline characteristics were summarized using standard descriptive statistics: frequencies and percentages for categorical variables while mean and standard deviation (SD) or standard error of mean (SEM) for continuous variables. Duration of follow-up was calculated from the date of the surgery to the date of progression (or date of last relevant clinical follow-up for those without progression), and from the date of the surgery to the date of death (or date of last known vital status for those not deceased). Progression-free survival (PFS) and overall survival (OS) were estimated using the Kaplan-Meier method. Univariate Cox proportional hazards models were fit to evaluate the association of each continuously-scaled marker with progression or death, respectively. Additional models 
were fit adjusting for stage (FIGO stage III/IV vs. I/II). Associations were summarized using the hazard ratio per 10 unit increase in each marker and corresponding 95\% confidence interval (CI) estimated from the models. Data were analysed using the SAS version 9.4 software package. $p$ Values were two-sided, with $p<0.05$ considered as significant.

\section{Bioinformatics analysis of TCGA database}

Biomarkers with statistically significant associations with both progression and death, after adjusting for stage, were further investigated through an external dataset. Uterine Corpus Endometrial Carcinoma (UCEC) data were retrieved from the publicly available and curated database cBioPortal (cBioPortal for cancer genomics, www.cbiop ortal.org, 2020), extracting clinical and gene expression features from the TCGA PanCancer Atlas dataset (Cerami et al. 2012; Gao et al. 2013). In particular, among the 529 cases, only 109 Uterine Serous Carcinoma/Uterine Papillary Serous Carcinoma cases were available. Gene expression levels were obtained from the mRNA Expression RSEM (Batch normalized from Illumina HiSeq RNASeqV2) dataset and matched to the clinical features. The best cutoff for gene expression values was chosen based on the results of Cutoff Finder analyses implemented in $\mathrm{R}$ v3.6.1 software environment ( $\mathrm{R}$ Core Team, 2019). The best cutoff value was used to categorize patients into low- and high-expression value groups. The prognostic effect of the clinical and molecular parameters on the risk of death was evaluated using the Cox proportional hazard model. Statistical analysis was performed using Stata software (StataCorp. 2011. Stata Statistical Software: Release 12. College Station, TX: StataCorp LP). In addition, we used cBioPortal online platform to predict co-expressed genes with the investigated biomarkers among the 109 USC samples of the TCGA data set.

\section{Results}

During January 2009 through December 2012, a total of 93 consecutive patients diagnosed with serous or mixed serous EC underwent primary surgery at our institution and met the following inclusion criteria: research authorization, no synchronous cancer or previous neoadjuvant therapies. Of these patients, 34 had mixed serous histology and 59 had serous histology. Among these 59 women, the results herein are based on the 36 patients whose specimens were available and there was adequate tissue in the paraffin block to analyze multiple markers with immunohistochemistry.

\section{Clinicopathological characteristics}

Clinicopathological characteristics of the 36 patients are summarized in Table 1. Mean age of patients at the time of surgery was 72.2 years (SD 9.0; 54.9-89.8 years). Half $(50 \%)$ of the patients had advanced stage of disease (from FIGO stage IIIA to stage IVB). Tumour diameter, available for 34 patients, was $>2 \mathrm{~cm}$ in $91.2 \%$ of cases; lymphvascular space invasion (LVSI) was present in six patients (16.7\%). More than two-third of patients presented myometrial invasion ( 29 patients, $80.6 \%$ ). Of the 30 patients who had a pelvic lymphadenectomy performed, $10(33.3 \%)$ had positive pelvic nodes. Of the 30 patients who had a paraaortic lymphadenectomy, 6 (20.0\%) had positive para-aortic nodes. Twenty-four $(66.4 \%)$ patients underwent any adjuvant treatment.

\section{Clinical outcomes}

During the follow-up period, progression and death of disease were observed in 18 and 23 patients, respectively. Based on the Kaplan-Meier method the median PFS and OS was 3.3 and 5.0 years, respectively. The median duration of follow-up was 5.3 years (IQR 0.4-7.4 years) among those without a progression and 7.4 years (IQR, 6.9-8.1 years) among the non-deceased patients.

The risk of progression was significantly higher among patients with advanced stage (III or IV) disease with a hazard ratio of 8.59 (95\% CI 2.62-28.23; $p<0.001$, Fig. 1a). In addition, the risk of death (due to any cause) was significantly higher among patients with advanced stage (III or IV) disease with a hazard ratio of 3.65 (95\% CI 1.49-8.94; $p=0.005$, Fig. 1b).

\section{Expression of molecular markers in the study cohort}

Figure 2 illustrates the mean percentage of expression (with SEM) for each marker in the nuclear and/or cytoplasmic cellular compartments. Accurate immunohistochemical analysis of tissue sections showed that FBXW7, Cyclin E and PLK1 were expressed in all samples and almost exclusively localized in the nuclear compartment. PLK1 is an essential mitotic kinase regulating multiple aspects of the cell division process. Activation of PLK1, driving cells into mitosis, requires phosphorylation of a conserved threonine residue (Thr 210), a process mainly mediated by Aurora A (Bruinsma et al. 2017); p(T210)PLK1 was expressed in the nuclear compartment of 23 out of 36 samples, this suggesting differences in pathway activation among patients. On the other hand, staining of Aurora A and active Aurora A phosphorylated on Thr288 was found in all samples in the nuclear and/or cytoplasmic compartments. Immunohistochemistry also showed that the expression of pGSK3 $\beta$, 
Table 1 Clinicopathological characteristics of uterine serous carcinomas (USC) patients

\begin{tabular}{|c|c|}
\hline Characteristics $^{\dagger}$ & Result \\
\hline All cases & 36 \\
\hline \multicolumn{2}{|l|}{ Age at surgery (years) } \\
\hline Mean (SD) & $72.2(9.0)$ \\
\hline Range & $54.8-89.8$ \\
\hline \multicolumn{2}{|l|}{ BMI $\left(\mathrm{kg} / \mathrm{m}^{2}\right)$} \\
\hline Mean (SD) & $32.8(8.9)$ \\
\hline Range & $15.8-60.5$ \\
\hline \multicolumn{2}{|l|}{ Surgical approach } \\
\hline Laparotomy & $28(77.8 \%)$ \\
\hline Robotic & $6(16.7 \%)$ \\
\hline Vaginal/robotic & $1(2.8 \%)$ \\
\hline Vaginal & $1(2.8 \%)$ \\
\hline \multicolumn{2}{|l|}{ FIGO stage } \\
\hline IA & $15(41.7 \%)$ \\
\hline IB & $2(5.6 \%)$ \\
\hline II & $1(2.8 \%)$ \\
\hline IIIA/B & $1(2.8 \%)$ \\
\hline IIIC1 & $5(13.9 \%)$ \\
\hline IIIC2 & $5(13.9 \%)$ \\
\hline IV & $7(19.4 \%)$ \\
\hline \multicolumn{2}{|l|}{ Tumour diameter } \\
\hline$\leq 2 \mathrm{~cm}$ & $3 / 34(8.8 \%)$ \\
\hline$>2 \mathrm{~cm}$ & $31 / 34(91.2 \%)$ \\
\hline \multicolumn{2}{|l|}{ LVSI } \\
\hline No & $30(83.3 \%)$ \\
\hline Yes & $6(16.7 \%)$ \\
\hline \multicolumn{2}{|l|}{ Myometrial invasion } \\
\hline No & $7(19.4 \%)$ \\
\hline Yes & $29(80.6 \%)$ \\
\hline \multicolumn{2}{|l|}{ Peritoneal cytology } \\
\hline Positive & $7 / 33(21.2 \%)$ \\
\hline Negative & $26 / 33(78.8 \%)$ \\
\hline \multicolumn{2}{|l|}{ Lymphadenectomy } \\
\hline No & $5(13.9 \%)$ \\
\hline Pelvic and para-aortic & $29(80.6 \%)$ \\
\hline Pelvic only & $1(2.8 \%)$ \\
\hline Paraaortic only & $1(2.8 \%)$ \\
\hline \multicolumn{2}{|l|}{ Positive lymph nodes } \\
\hline Pelvic positive & $10 / 30(33.3 \%)$ \\
\hline Paraaortic positive & $6 / 30(20.0 \%)$ \\
\hline \multicolumn{2}{|l|}{ Adjuvant therapy } \\
\hline None & $7(19.4 \%)$ \\
\hline VBT only & $8(22.2 \%)$ \\
\hline Chemo \pm VBT & $7(19.4 \%)$ \\
\hline $\mathrm{Chemo} \pm \mathrm{EBRT} \pm \mathrm{VBT}$ & $9(25.0 \%)$ \\
\hline Unknown & $5(13.9 \%)$ \\
\hline
\end{tabular}

$B M I$ body mass index, LVSI lymphovascular space invasion, VBT vaginal brachytherapy, $E B R T$ external beam radiation therapy

${ }^{\dagger}$ Results reported as $N(\%)$ unless otherwise noted
FOXM1, PPP2R1B and CIP2A was predominantly evident in the cytoplasm of all analysed sections. It is worth noting that FOXM1, a critical proliferation-associated transcription factor closely involved with the processes of cell proliferation, self-renewal and tumorigenesis (Liao et al. 2018) was present as nuclear staining in all patients, although at a different extent among samples.

Expression of E2F-1 was exclusively nuclear, with low activation, as demonstrated by the negative/low levels of $\mathrm{p}(\mathrm{S} 364) \mathrm{E} 2 \mathrm{~F}-1$ detected in the majority of the sections. Most samples were negative for p73 (21 out of 36); however, when p73 was expressed, the protein was localized in the nucleus. Cyclin B1 was mainly localized into the cytoplasmic compartment, although a low nuclear staining was also evident in most samples.

All sections analysed showed intense CHK1 staining in the cytoplasmic localization, with the majority also exhibiting low-medium nuclear expression. Finally, low/medium BRCA1 and RAD51 levels were detected in most sections.

\section{Associations between biomarker levels and clinical outcomes}

The association of each biomarker with the risk of recurrence and death, respectively, was evaluated univariately and after adjusting for stage (i.e. FIGO stage III/IV vs. I/ II). The hazard ratios (per 10-unit increase) are presented in Tables 2and 3. Consistently high-expression levels of CIP2A were detected in all samples and, therefore, this protein was not evaluated in this analysis. Likewise, due to the low number of positive samples (8 out of 36), p(S364) E2F-1 was also not included in outcome analysis.

Focusing on the analysis adjusted for stage, we found that a higher expression of CHK1 both in the nuclear and cytoplasmic compartments was significantly associated with a decreased risk of progression $(\mathrm{HR}=0.57$ and 0.56 , respectively). Likewise, higher nuclear FBXW7 and cytoplasmic PPP2R1B levels were significantly associated with a decreased risk of progression ( $\mathrm{HR}=0.80$ and 0.59 , respectively). CHK1 was the only biomarker significantly associated with death, and patients with high protein expression showed a reduced risk of death ( $\mathrm{HR}=0.75$ and 0.66 , for nuclear and cytoplasmic localization, respectively). Finally, cyclin B1, when overexpressed in the nucleus, was shown to be a negative prognostic factor for death $(\mathrm{HR}=1.55$, $p=0.07$ ).

Interestingly higher nuclear BRCA1 expression was associated with an increased risk of progression $(\mathrm{HR}=1.47$, $p=0.025)$ and death $(\mathrm{HR}=1.31, p=0.06)$ upon univariate analysis. However, after adjusting for stage, both of these associations were greatly attenuated $(\mathrm{HR}=1.08, p=0.92$; $\mathrm{HR}=1.09, p=0.59$ ). 

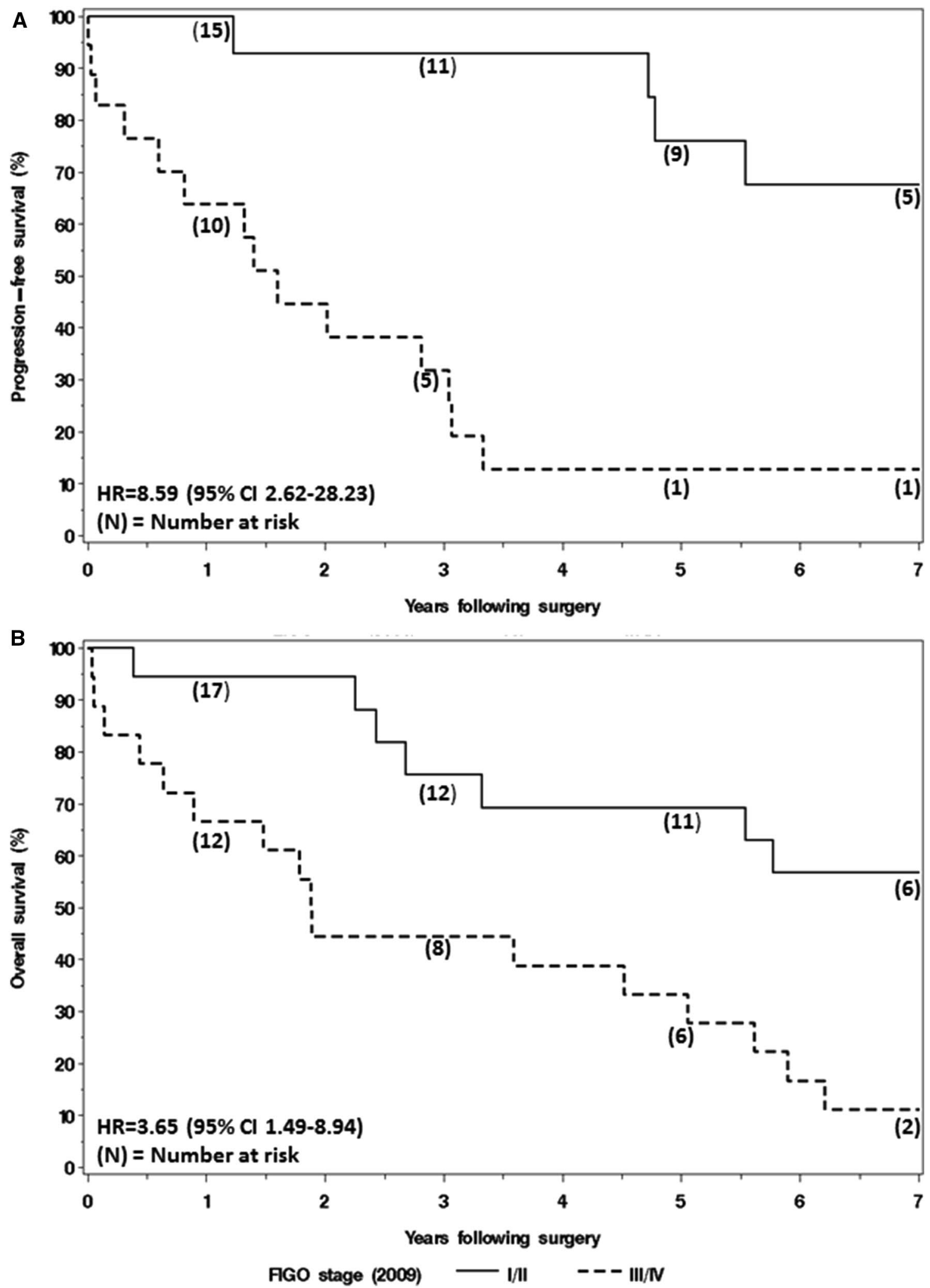

Fig. 1 Kaplan-Meier curves for progression-free survival (a) and overall survival (b), according to stage 
Fig. 2 Immunohistochemistrybased protein profiling of uterine serous carcinomas (UCS) patients. Bar charts show protein expression grouped in PI3K-AKT-FBXW7 pathway (a), E2F-1-mediated pathway (b) and HR-related proteins (c). The bars represent the mean \pm SEM of positive cells for the indicated proteins in the nuclear (nuc) and/or cytoplasmic (cyt) compartment $(n=36)$. $P$ indicates the phosphorylated form of the protein. Adjacent to each graph are shown representative immunohistochemical pictures of proteins significantly affecting PFS or OS when adjusting for stage (magnification 20X)
A

\section{PI3K-AKT-FBXW7 pathway}

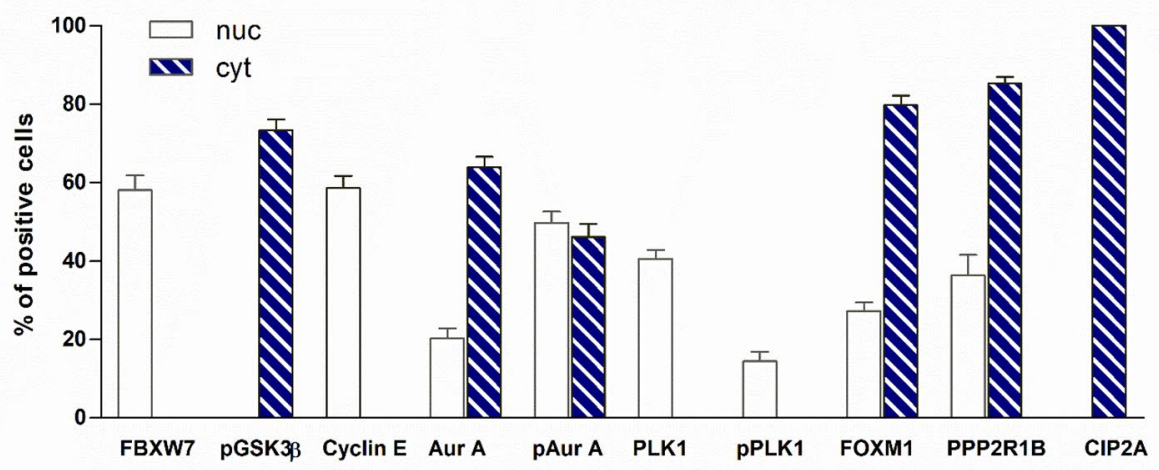

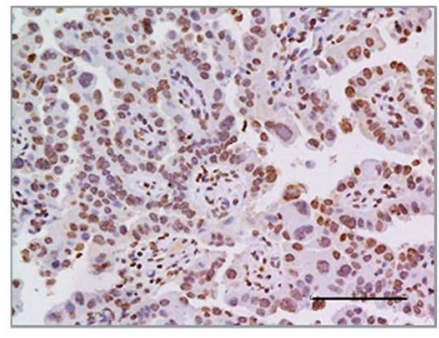

FBXW7

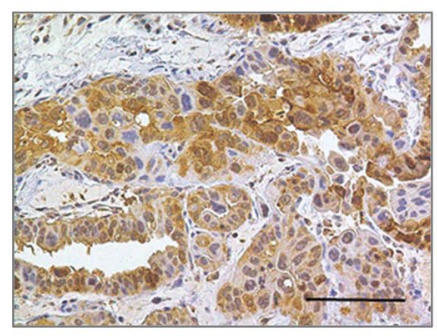

PPP2R1B
B

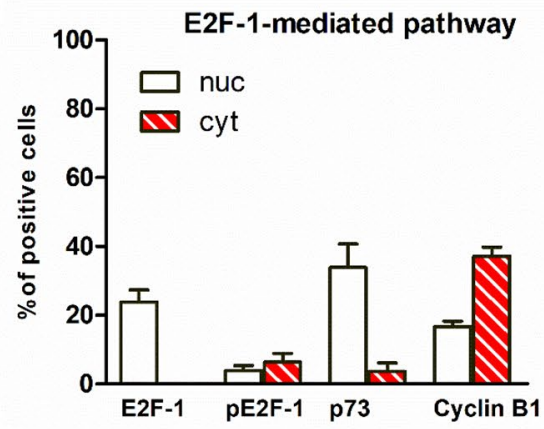

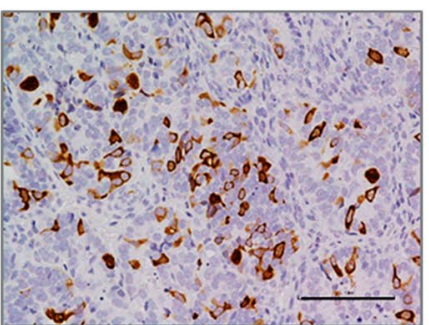

Cyclin B1
C

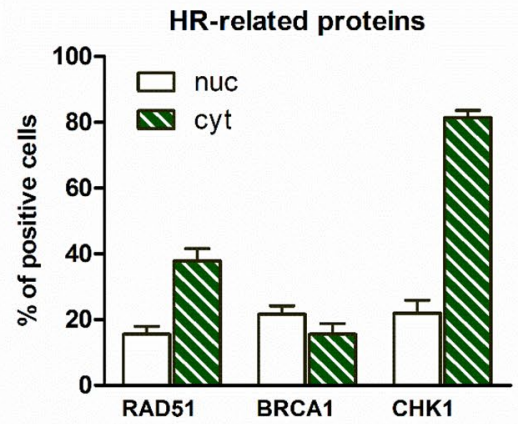

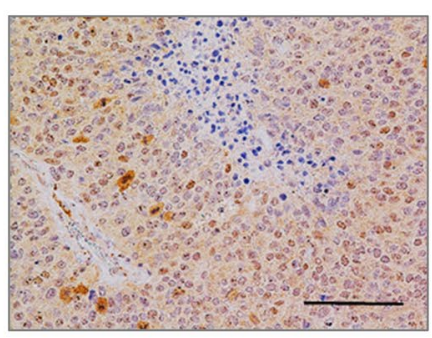

CHK1

\section{Association of CHK1 expression to survival prognosis in TCGA cohort}

To find confirmation for CHK1 as a favourable marker of outcome in USC patients, we interrogated the TCGA dataset by evaluating the association between CHEK1 (CHK1) mRNA levels and the risk of death in data from 109 patients, this latter being the first recommended clinical outcome endpoint for USC (Liu et al. 2018). We found that, after adjusting for tumour stage, CHK1 expression > 379.2 ("high CHK1") was 
Table 2 Univariate analysis of markers evaluated for an association with progression in uterine serous carcinomas (USC) patients

\begin{tabular}{|c|c|c|c|c|}
\hline \multirow[t]{2}{*}{ Marker } & \multicolumn{2}{|c|}{$\begin{array}{l}\text { Univariate analysis, } \\
\text { unadjusted }\end{array}$} & \multicolumn{2}{|c|}{$\begin{array}{l}\text { Univariate analysis, } \\
\text { adjusted for stage }\end{array}$} \\
\hline & $\operatorname{HR}(95 \%$ CI $)$ & $P^{\ddagger}$ & HR (95\% CI) & $P^{\ddagger}$ \\
\hline FBXW7 nuc & $0.85(0.71,1.01)$ & 0.07 & $0.80(0.66,0.97)$ & 0.024 \\
\hline pGSK3 $\beta$ cyt & $1.01(0.74,1.37)$ & 0.96 & $1.09(0.75,1.57)$ & 0.67 \\
\hline Cyclin E nuc & $0.96(0.77,1.19)$ & 0.69 & $1.05(0.85,1.29)$ & 0.64 \\
\hline Aurora A nuc & $1.14(0.82,1.59)$ & 0.44 & $0.89(0.65,1.22)$ & 0.46 \\
\hline Aurora A cyt & $1.29(0.97,1.72)$ & 0.08 & $1.22(0.91,1.64)$ & 0.18 \\
\hline pAurora A nuc & $0.93(0.73,1.20)$ & 0.59 & $0.92(0.66,1.29)$ & 0.63 \\
\hline pAurora A cyt & $1.07(0.83,1.38)$ & 0.60 & $0.99(0.78,1.25)$ & 0.91 \\
\hline PLK1 nuc & $0.77(0.50,1.18)$ & 0.23 & $0.97(0.62,1.54)$ & 0.91 \\
\hline pPLK1 nuc & $0.86(0.60,1.23)$ & 0.41 & $0.82(0.55,1.20)$ & 0.30 \\
\hline FOXM1 nuc & $1.03(0.71,1.48)$ & 0.90 & $1.02(0.71,1.46)$ & 0.92 \\
\hline FOXM1 cyt & $1.39(0.99,1.94)$ & 0.06 & $1.26(0.89,1.77)$ & 0.19 \\
\hline PPP2R1B nuc & $0.93(0.80,1.10)$ & 0.40 & $0.92(0.77,1.10)$ & 0.37 \\
\hline PPP2R1B cyt & $0.85(0.53,1.36)$ & 0.49 & $0.59(0.36,0.96)$ & 0.035 \\
\hline E2F-1 nuc & $0.89(0.71,1.12)$ & 0.32 & $0.78(0.58,1.05)$ & 0.11 \\
\hline p73 nuc & $1.02(0.90,1.14)$ & 0.80 & $1.00(0.89,1.12)$ & 0.97 \\
\hline Cyclin B1 nuc & $1.04(0.63,1.70)$ & 0.89 & $1.44(0.82,2.51)$ & 0.20 \\
\hline Cyclin B1 cyt & $0.80(0.56,1.13)$ & 0.21 & $0.88(0.59,1.32)$ & 0.54 \\
\hline RAD51 cyt & $0.91(0.74,1.11)$ & 0.34 & $0.87(0.69,1.10)$ & 0.25 \\
\hline RAD51 nuc & $0.97(0.69,1.36)$ & 0.87 & $0.91(0.63,1.31)$ & 0.61 \\
\hline BRCA1 nuc & $1.48(1.05,2.09)$ & 0.025 & $1.08(0.73,1.60)$ & 0.72 \\
\hline BRCA1 cyt & $1.12(0.89,1.41)$ & 0.35 & $0.93(0.74,1.17)$ & 0.53 \\
\hline CHK1 nuc & $0.67(0.50,0.90)$ & 0.008 & $0.57(0.39,0.82)$ & 0.002 \\
\hline CHK1 cyt & $0.91(0.66,1.26)$ & 0.57 & $0.56(0.36,0.87)$ & 0.010 \\
\hline
\end{tabular}

$P F S$ progression-free survival, $H R$ hazard ratio per 10 unit increase in each marker, $C I$ confidence interval, $N u c$ nuclear compartment, $C y t$ cytoplasmic compartment

${ }^{\dagger}$ Stage was categorized as III/IV vs. I/II when included as a covariate in each regression model

${ }^{\ddagger} P$ values were derived from the Cox proportional hazards regression model

associated with a decrease in the risk of death (HR 0.35, 95\% CI $0.16-0.77, p=0.01)$. To gain insights into the signalling triggered by CHK1, we used cBioPortal (http://www.cbiop ortal.org/) to predict CHK1 co-expression genes among the 109 USC samples of the TCGA data set. Interestingly, we found that the protein-coding gene identified with the highest Spearman correlation value was EI24 (etoposide-induced gene 2.4) (Spearman's correlation $=0.72, p<0.001$ ). This gene has been shown to play an important role in negative cell growth control, apoptosis and activation of autophagy (Zhao et al. 2012).
Table 3 Univariate analysis of markers evaluated for an associations with death (due to any cause) in uterine serous carcinomas (USC) patients

\begin{tabular}{llllll}
\hline Marker & \multicolumn{2}{l}{$\begin{array}{l}\text { Univariate analysis, } \\
\text { unadjusted }\end{array}$} & & \multicolumn{2}{l}{$\begin{array}{l}\text { Univariate analysis, } \\
\text { adjusted for stage }\end{array}$} \\
\cline { 2 - 3 } \cline { 5 - 6 } & HR $(95 \%$ CI $)$ & $P^{\ddagger}$ & & HR $(95 \%$ CI $)$ & $P^{\ddagger}$ \\
\hline FBXW7 nuc & $0.93(0.79,1.10)$ & 0.38 & & $0.94(0.79,1.11)$ & 0.44 \\
pGSK3 $\beta$ cyt & $0.85(0.65,1.10)$ & 0.22 & & $0.80(0.60,1.06)$ & 0.12 \\
Cyclin E nuc & $0.95(0.78,1.16)$ & 0.63 & & $1.00(0.83,1.21)$ & 0.98 \\
Aurora A nuc & $1.43(1.09,1.89)$ & 0.011 & & $1.25(0.96,1.64)$ & 0.10 \\
Aurora A cyt & $1.13(0.88,1.44)$ & 0.34 & & $1.00(0.78,1.28)$ & 0.99 \\
pAurora A nuc & $0.93(0.74,1.17)$ & 0.54 & & $0.93(0.71,1.21)$ & 0.57 \\
pAurora A cyt & $1.05(0.85,1.31)$ & 0.63 & & $1.02(0.85,1.24)$ & 0.81 \\
PLK1 nuc & $0.80(0.56,1.16)$ & 0.24 & & $0.97(0.66,1.44)$ & 0.89 \\
pPLK1 nuc & $0.98(0.73,1.33)$ & 0.92 & & $1.03(0.76,1.40)$ & 0.84 \\
FOXM1 nuc & $0.82(0.58,1.14)$ & 0.24 & & $0.78(0.56,1.08)$ & 0.13 \\
FOXM1 cyt & $1.08(0.83,1.41)$ & 0.57 & & $0.96(0.73,1.26)$ & 0.75 \\
PPP2R1B nuc & $1.00(0.88,1.14)$ & 0.97 & & $1.02(0.89,1.17)$ & 0.78 \\
PPP2R1B cyt & $1.06(0.66,1.70)$ & 0.80 & & $0.82(0.51,1.30)$ & 0.39 \\
E2F-1 nuc & $0.90(0.74,1.10)$ & 0.29 & & $0.84(0.66,1.06)$ & 0.13 \\
p73 nuc & $1.07(0.96,1.18)$ & 0.21 & & $1.04(0.94,1.15)$ & 0.47 \\
Cyclin B1 nuc & $1.18(0.76,1.82)$ & 0.46 & & $1.55(0.96,2.50)$ & 0.07 \\
Cyclin B1 cyt & $0.75(0.54,1.03)$ & 0.08 & & $0.76(0.53,1.08)$ & 0.13 \\
RAD51 cyt & $0.89(0.74,1.07)$ & 0.21 & & $0.87(0.71,1.06)$ & 0.17 \\
RAD51 nuc & $1.06(0.79,1.41)$ & 0.72 & & $1.07(0.79,1.44)$ & 0.67 \\
BRCA1 nuc & $1.31(0.98,1.74)$ & 0.06 & & $1.09(0.79,1.50)$ & 0.59 \\
BRCA1 cyt & $1.07(0.89,1.30)$ & 0.48 & & $0.91(0.74,1.12)$ & 0.38 \\
CHK1 nuc & $0.76(0.61,0.94)$ & 0.013 & & $0.75(0.59,0.96)$ & 0.023 \\
CHK1 cyt & $0.92(0.68,1.23)$ & 0.56 & & $0.66(0.46,0.96)$ & 0.028 \\
\hline
\end{tabular}

$O S$ overall survival, $H R$ hazard ratio per 10 unit increase in each marker, $C I$ confidence interval, $N u c$ nuclear compartment, $C y t$ cytoplasmic compartment

${ }^{\dagger}$ Stage was categorized as III/IV vs. I/II when included as a covariate in each regression model

${ }^{\ddagger} p$ Values were derived from the Cox proportional hazards regression model

\section{Discussion}

Results from the present study offer some important insights into USC, which is a rare aggressive subtype of endometrial cancer (Brooks et al. 2019).

The most interesting finding emerging from our investigation is the favourable prognostic role of CHK1 for both progression-free and overall survival, a result consistently observed for both the nuclear and the cytoplasmic protein fraction. Interestingly, analysis of a TCGA data set of 109 USC patients corroborates our results showing a favourable prognostic role of CHK1 after adjusting for stage. CHK1 is a key signal transducer in the DNA-damage response pathways, being implicated in the induction of cell-cycle arrest, DNA repair and apoptosis (Bartek and Lukas 2003). The 
protein is mainly expressed in the nucleus, but, following activation, it shuttles between the nucleus and the cytoplasm, regulating both nuclear and cytoplasmic checkpoints; importantly, cytoplasmic CHK1 does not support cell viability (Wang et al. 2012). Uncertainty exists in the literature about the role of CHK1 in cancer development and progression. Indeed, given the regulatory role it plays in DNA damage, CHK1 has long been recognized as a tumour suppressor. However, recent evidences indicate that CHK1 may contribute to tumour growth, thus representing a potential target in anti-cancer therapy. The notion that tumour cells (often p53-deficient and defective in G1 arrest), unlike normal cells, rely mainly on $\mathrm{S}$ or $\mathrm{G} 2$ checkpoints mediated by CHK1 to repair their damaged DNA and preserve their genomic integrity for basic viability, supports this view (Chen et al. 2006). In line with this concept, no homozygous loss-offunction mutation of CHK1 has been detected in a wide range of human tumours (Remmerie and Janssens 2019), an observation suggesting that cells with defective CHK1 are eliminated during tumorigenesis. Finally, the gene has been found overexpressed in variety of tumours and expression correlated with tumour grade and disease recurrence (Zhang and Hunter 2014). On the other hand, CHK1 frameshift mutations have been reported in genetically unstable colorectal and endometrial cancers, and these mutations might be involved in tumorigenesis, through a defect in response to DNA damage (Bertoni et al. 1999; Vassileva et al. 2002). Although these mutations predict for truncated CHK1 protein, their pathogenic implication is not evident since they have been always found as heterozygous aberrations. In keeping with our results, previous studies have also demonstrated that constitutive activation of CHK1, in the absence of DNA-damage, leads to cell-cycle arrest and eventually cell death (Wang et al. 2012; Zhang and Hunter 2014). Indeed, by querying the public TCGA USC sequencing data, we found that the top coding-gene associated with CHK1 was EI24, a putative tumour suppressor gene, whose reduced expression has been associated with the induction of EMT and tumour progression (Choi et al. 2013). Notably, EI24 has been also characterized as an E2F-1 target gene (Sung et al. 2013). Relevant to our results, Urist and colleagues (2004) demonstrated that CHK1 is required for induction of p73 following DNA damage and that E2F-1 is critical in this regulation. This implies that the CHK1-E2F-1-p73 pathway is central to $\mathrm{p} 53$-independent apoptosis, after DNA damage. Interestingly, literature data also unveiled Aurora A tumorigenic properties actually mediated by repression of CHK1 kinase activity, with a consequent impairment of the error-free homologous recombination pathway (Sourisseau et al. 2010). Overall, these findings might support the idea that in USC elevated constitutive levels of CHK1 play a protective role in disease development, through induction of permanent cell-cycle arrest and cell death. In addition, the outcome of CHK1 signalling following DNA-damaging therapies may also lead to increased apoptosis linked to $\mathrm{p} 73$ induction.

Besides CHK1, also FBXW7 and PPP2R1B were found to have a favourable prognostic role for progression, in analysis adjusted for stage. FBXW7 is a candidate driver gene somatically mutated in about 15-29\% USC (Le Gallo et al. 2012; Cancer Genome Atlas Research Network 2013). Besides its role as tumour suppressor, emerging evidence suggest that lack of protein or loss-of-function mutations in FBXW7 confer resistance to antitubulin agents (Wertz et al. 2011), while sensitizing to HDAC inhibitors (Garnett et al. 2012). In this context, results of our study, if confirmed in a larger population, could have a clinical significance in guiding personalized therapy. With regard to PPP2R1B, our findings are in line with available data testifying a regulator function in a variety of cellular processes, including cell-cycle progression, whose alteration may be involved in tumorigenesis (Calin et al. 2000). However, to the best of our knowledge, no other evidences are available on its role as prognostic biomarkers in USC. Again, these findings could open new perspective and potential strategies for drug target discovery in USC therapy.

Results from the present study also suggest that Cyclin $\mathrm{B} 1$ is an unfavourable prognostic biomarker for death in USC. Cyclin B1 is a positive regulator of cell-cycle progression; protein overexpression reduces cell-cycle length and enables cells to override the G2 DNA damage checkpoint (Jin et al. 1998; Pomerening et al. 2005), finally leading to uncontrolled proliferation. At present, there are few studies regarding the role of Cyclin B1 in USC development and progression, although recent findings by Kwan and colleagues (Kwan et al. 2020) showed high Cyclin B1 expression levels in USC.

Finally, we also found that, at univariate analysis, higher nuclear BRCA1 expression was associated with an increased risk of progression and death. This result is in line with data from HGSOC, showing that patients with low BRCA1 expression had a more favourable outcome (Weberpals et al. 2011).

Beside the above-mentioned biomarkers, here we also give evidence that, although not having a role as prognostic factors, some proteins are expressed at high levels in USC tumours and specific pathways activated. In this respect, our findings therefore also provide hints for further studies specifically investigating those proteins found to be highly expressed in the disease and their potential value as new therapeutic targets.

Among the peculiar strengths of this study are that patients were managed in a tertiary centre, with well-documented electronic charts and dedicated personnel drawing up the historical EC database. In addition, the follow-up was sufficiently long to capture enough events, thus providing adequate statistical 
power to identify meaningful associations. Finally, all samples were treated and analysed with standard protocols. Besides the above-mentioned strengths, few limitations in our study need to be acknowledged. First, sample size was not large enough for detecting modest associations between biomarkers expression and the risk of progression or death. Moreover, pathologist-dependent interpretation of immunohistochemical staining might represent a setback, therefore, requiring all necessary steps to avoid any bias. Not least important, the retrospective nature of such a study design can lead to missing data.

In conclusion, results from the present study, although preliminary, highlight that molecular tumour profiling may provide a prognostic tool in serous endometrial cancer, representing an essential first step in drug discovery and development of personalized therapy.

Supplementary Information The online version contains supplementary material available at https://doi.org/10.1007/s00432-021-03566-x.

Acknowledgements We thank Doctor Gabriele Babini for bioinformatic analysis.

Author contributions Conception and design: GD, AM, GS and DG. Provision of study material or patients: AM, GV and PZA. Methodology and investigation: EM and AC. Pathologic and immunohistochemical analysis: GFZ and EM. Data collection and interpretation: GD, FF and DG. Statistical analysis: AW. Bioinformatic analysis: GB. Writing original draft: GD, AW and GB. Writing review and editing: AM, GS and DG. Supervision: AM, GS and DG. Funding acquisition: GS. Final approval of manuscript: All authors. Accountable for all aspects of the work: All authors.

Funding Open access funding provided by Università Cattolica del Sacro Cuore within the CRUI-CARE Agreement. This work, as part of the activities of the Unit of Translational Medicine for Woman and Child Health, was partially supported by the "Associazione OPPO e le sue stanze" Onlus.

\section{Compliance with ethical standards}

Conflict of interest The authors have no conflict of interest or potential financial disclosures.

Availability of data and material The data that support the findings of this study are available from the corresponding author, (GS), upon request.

Ethics approval Written informed consent was obtained from the participants of the study.

Consent to participate Informed consent was obtained from all individual participants included in the study.

Consent for publication Consent for publication was obtained from all individual participants.

Open Access This article is licensed under a Creative Commons Attribution 4.0 International License, which permits use, sharing, adaptation, distribution and reproduction in any medium or format, as long as you give appropriate credit to the original author(s) and the source, provide a link to the Creative Commons licence, and indicate if changes were made. The images or other third party material in this article are included in the article's Creative Commons licence, unless indicated otherwise in a credit line to the material. If material is not included in the article's Creative Commons licence and your intended use is not permitted by statutory regulation or exceeds the permitted use, you will need to obtain permission directly from the copyright holder. To view a copy of this licence, visit http://creativecommons.org/licenses/by/4.0/.

\section{References}

Alkushi A, Clarke BA, Akbari M, Makretsov N, Lim P, Miller D et al (2007) Identification of prognostically relevant and reproducible subsets of endometrial adenocarcinoma based on clustering analysis of immunostaining data. Mod Pathol 20:1156-1165. https://doi.org/10.1038/modpathol.3800950

Bartek J, Lukas J (2003) Chk1 and Chk2 kinases in checkpoint control and cancer. Cancer Cell 3:421-429. https://doi.org/10.1016/ s1535-6108(03)00110-7

Bertoni F, Codegoni AM, Furlan D, Tibiletti MG, Capella C, Broggini M (1999) CHK1 frameshift mutations in genetically unstable colorectal and endometrial cancers. Genes Chromosomes Cancer 26:176-180. https://doi.org/10.1007/s00018-013-1307-3

Bokhman JV (1983) Two pathogenetic types of endometrial carcinoma. Gynecol Oncol 15:10-17. https://doi.org/10.1016/00908258(83)90111-7

Brooks RA, Fleming GF, Lastra RR, Lee NK, Moroney JW, Son CH et al (2019) Current recommendations and recent progress in endometrial cancer. CA Cancer J Clin 69(4):258-279. https:// doi.org/10.3322/caac.21561

Bruinsma W, Aprelia M, García-Santisteban I, Kool J, Xu YJ, Medema RH (2017) Inhibition of Polo-like kinase 1 during the DNA damage response is mediated through loss of Aurora A recruitment by Bora. Oncogene 36:1840-1848. https://doi.org/ 10.1038/onc. 2016.347

Calin G, Di Iasio M, Caprini E, Vorechovsky NPG, Sozzi G et al (2000) Low frequency of alterations of the $\alpha$ (PPP2R1A) and $\beta$ (PPP2R1B) isoforms of the subunit A of the serine-threonine phosphatase 2A in human neoplasms. Oncogene 19:1191-1195. https://doi.org/10.1038/sj.onc.1203389

Cancer Genome Atlas Research Network, Kandoth C, Schultz N, Cherniack AD, Akbani R, Liu Y, Shen H et al (2013) Integrated genomic characterization of endometrial carcinoma. Nature 497(7447):67-73. https://doi.org/10.1038/nature12113 (published correction appears in Nature. 2013 Aug 8; 500 (7461):242)

cBioPortal for cancer genomics, www.cbioportal.org, 2020 (accessed 1 June 2020).

Cerami E, Gao J, Dogrusoz U, Gross BE, Sumer SO, Aksoy BA et al (2012) The cBio cancer genomics portal: an open platform for exploring multidimensional cancer genomics data. Cancer Discov 2:401-404. https://doi.org/10.1158/2159-8290.CD-12-0095

Chen Z, Xiao Z, Gu WZ, Xue J, Bui MH, Kovar P et al (2006) Selective Chk1 inhibitors differentially sensitize p53-deficient cancer cells to cancer therapeutics. Int J Cancer 119:2784-2794. https://doi.org/10.1002/ijc.22198

Choi JM, Devkota S, Sung YH, Lee HW (2013) Lee EI24 regulates epithelial-to-mesenchymal transition and tumor progression by suppressing TRAF2-mediated NF-kappaB activity. Oncotarget 4:2383-2396. https://doi.org/10.18632/oncotarget.1434 
De Jonge MM, Auguste A, van Wijk LM, Schouten PC, Meijers M, Ter Haar NT et al (2019) Frequent homologous recombination deficiency in high-grade endometrial carcinomas. Clin Cancer Res 25:1087-1097. https://doi.org/10.1158/1078-0432. CCR-18-1443

Dedes KJ, Wetterskog D, Ashworth A, Kaye SB, Reis-Filho JS (2011) Emerging therapeutic targets in endometrial cancer. Nat Rev Clin Oncol 8:261-271. https://doi.org/10.1038/nrclinonc.2010.216

Del Carmen MG, Birrer M, Schorge JO (2012) Uterine papillary serous cancer: a review of the literature. Gynecol Oncol 127:651-661. https://doi.org/10.1016/j.ygyno.2012.09.012

Galindo-Moreno M, Giráldez S, Limón-Mortés MC, Belmonte-Fernández A, Sáez C, Japón MÁ et al (2020) p53 and FBXW7: sometimes two guardians are worse than one. Cancers (Basel) 12:985. https://doi.org/10.3390/cancers12040985

Gao J, Aksoy BA, Dogrusoz U, Dresdner G, Gross B, Sumer SO et al (2013) Integrative analysis of complex cancer genomics and clinical profiles using the cBioPortal. Sci Signal 6(269):11. https://doi. org/10.1126/scisignal.2004088

Garnett M, Edelman E, Heidorn S, Greenman CD, Dastur A, Lau KW et al (2012) Systematic identification of genomic markers of drug sensitivity in cancer cells. Nature 483:570-575. https://doi.org/ 10.1038/nature11005

Gentry-Maharaj A, Karpinskyj C (2020) Current and future approaches to screening for endometrial cancer. Best Pract Res Clin Obstet Gynaecol 65:79-97. https://doi.org/10.1016/j.bpobgyn.2019.12. 006

Hallstrom TC, Mori S, Nevins JR (2008) An E2F1-dependent gene expression program that determines the balance between proliferation and cell death. Cancer Cell 13:11-22. https://doi.org/10. 1016/j.ccr.2007.11.031

Hamilton CA, Cheung MK, Osann K, Chen L, Teng NN, Longacre TA et al (2006) Uterine papillary serous and clear cell carcinomas predict for poorer survival compared to grade 3 endometrioid corpus cancers. Br J Cancer 4:642-646. https://doi.org/10.1038/ sj.bjc. 6603012

Hamilton CA, Kapp DS, Chan JK (2008) Clinical aspects of uterine papillary serous carcinoma. Curr Opin Obstet Gynecol 20:26-33. https://doi.org/10.1097/GCO.0b013e3282f2b10d

Hoxhaj G, Manning BD (2020) The PI3K-AKT network at the interface of oncogenic signalling and cancer metabolism. Nat Rev Cancer 20:74-88. https://doi.org/10.1038/s41568-019-0216-7

Jin P, Hardy S, Morgan DO (1998) Nuclear localization of cyclin B1 controls mitotic entry after DNA damage. J Cell Biol 141:875885. https://doi.org/10.1083/jcb.141.4.875

Keys HM, Roberts JA, Brunetto VL, Zaino RJ, Spirtos NM, Bloss JD et al (2004) A phase III trial of surgery with or without adjunctive external pelvic radiation therapy in intermediate risk endometrial adenocarcinoma: a gynecologic oncology group study. Gynecol Oncol 92:744-751. https://doi.org/10.1016/j.ygyno.2003.11.048

Kuhn E, Wu RC, Guan B, Gang Wu, Zhang J, Wang Y et al (2012) Identification of molecular pathway aberrations in uterine serous carcinoma by genome-wide analyses. J Natl Cancer Inst 104:1503-1513. https://doi.org/10.1093/jnci/djs345

Kuo YC, Huang KY, Yang CH, Yang YS, Lee WY, Chiang CW (2008) Regulation of phosphorylation of Thr-308 of Akt, cell proliferation, and survival by the B55alpha regulatory subunit targeting of the protein phosphatase 2A holoenzyme to Akt. J Biol Chem 283:1882-1892. https://doi.org/10.1074/jbc.M709585200

Kwan SY, Au-Yeung CL, Yeung TL, Rynne-Vidal A, Wong K-K, Risinger JI et al (2020) Ubiquitin carboxyl-terminal hydrolase L1 (UCHL1) promotes uterine serous cancer cell proliferation and cell cycle progression. Cancers (Basel) 12:118. https://doi.org/ $10.3390 /$ cancers 12010118
Le Gallo M, O’Hara AJ, Rudd ML, Urick ME, Hansen NF, O'Neil $\mathrm{NJ}$ et al (2012) Exome sequencing of serous endometrial tumors identifies recurrent somatic mutations in chromatin-remodeling and ubiquitin ligase complex genes. Nat Genet 44:1310-1315. https://doi.org/10.1038/ng.2455

Liao GB, Li XZ, Zeng S, Liu C, Yang S-M, Yang L et al (2018) Regulation of the master regulator FOXM1 in cancer. Cell Commun Signal 16:57. https://doi.org/10.1186/s12964-018-0266-6

Liu J, Lichtenberg T, Hoadley KA, Poisson LM, Lazar AJ, Cherniack AD et al (2018) An integrated TCGA pan-cancer clinical data resource to drive high-quality survival outcome analytics. Cell 173(400-416):e11. https://doi.org/10.1016/j.cell.2018.02.052

Nout RA, Smit VT, Putter H, Jürgenliemk-Schulz IM, Jobsen JJ, Lutgens LCHW et al (2010) Vaginal brachytherapy versus pelvic external beam radiotherapy for patients with endometrial cancer of high-intermediate risk (PORTEC-2): an open-label, non-inferiority, randomised trial. Lancet 375(9717):816-823. https://doi. org/10.1016/S0140-6736(09)62163-2

Pomerening JR, Kim SY, Ferrell JE Jr (2005) Systems-level dissection of the cell-cycle oscillator: bypassing positive feedback produces damped oscillations. Cell 122:565-578. https://doi.org/10.1016/j. cell.2005.06.016

Remmerie M, Janssens V (2019) PP2A: a promising biomarker and therapeutic target in endometrial cancer. Front Oncol 9:462. https://doi.org/10.3389/fonc.2019.00462

Ring K, Bruegl A, Allen B, Elkin EP, Singh N, Hartman AR et al (2016) Germline multi-gene hereditary cancer panel testing in an unselected endometrial cancer cohort. Mod Pathol 29:1381-1389. https://doi.org/10.1038/modpathol.2016.135

R Development Core Team (2019). R: A Language and Environment for Statistical Computing. Vienna, Austria: R Foundation for Statistical Computing

Seeber LM, Zweemer RP, Marchionni L, Massuger LFAG, Smit VTHBM, Marchien van Baal W et al (2010) Methylation profiles of endometrioid and serous endometrial cancers. Endocr Relat Cancer 17:663-673. https://doi.org/10.1677/ERC-10-0014

Sourisseau T, Maniotis D, McCarthy A, Tang C, Lord CJ, Ashworth A et al (2010) Aurora-A expressing tumour cells are deficient for homology-directed DNA double strand-break repair and sensitive to PARP inhibition. EMBO Mol Med 2:130-142. https://doi.org/ 10.1002/emmm.201000068

Sung YH, Jin Y, Kang Y, Devkota S, Lee J, Roh J-I et al (2013) Ei24, a novel E2F target gene, affects p53-independent cell death upon ultraviolet C irradiation. J Biol Chem 288:31261-31267. https:// doi.org/10.1074/jbc.M113.477570

Urist M, Tanaka T, Poyurovsky MV, Prives C (2004) p73 induction after DNA damage is regulated by checkpoint kinases Chk 1 and Chk2. Genes Dev 18:3041-3054. https://doi.org/10.1101/gad. 1221004

Vassileva V, Millar A, Briollais L, Chapman W, Bapat B (2002) Genes involved in DNA repair are mutational targets in endometrial cancers with microsatellite instability. Cancer Res 62:4095-4099. https://doi.org/10.2147/AGG.S28953

Wang J, Han X, Feng X, Wang Z, Zhang Y (2012) Coupling cellular localization and function of checkpoint kinase 1 (Chk1) in checkpoints and cell viability. J Biol Chem 287:25501-25509. https:// doi.org/10.1074/jbc.M112.350397

Weberpals JI, Tu D, Squire JA, Amin MS, Islam S, Pelletier LB et al (2011) Breast cancer 1 (BRCA1) protein expression as a prognostic marker in sporadic epithelial ovarian carcinoma: an NCIC CTG OV.16 correlative study. Ann Oncol 22:2403-2410. https:// doi.org/10.1093/annonc/mdq770

Wertz I, Kusam S, Lam C, Okamoto T, Sandoval W, Anderson DJ et al (2011) Sensitivity to antitubulin chemotherapeutics is regulated 
by MCL1 and FBW7. Nature 471:110-114. https://doi.org/10. 1038/nature09779

Zhang Y, Hunter T (2014) Roles of Chk1 in cell biology and cancer therapy. Int J Cancer 134:1013-1023. https://doi.org/10.1002/ijc. 28226

Zhao YG, Zhao H, Miao L, Wang L, Sun F, Zhang H (2012) The p53-induced gene Ei24 is an essential component of the basal autophagy pathway. J Biol Chem 287:42053-42063. https://doi. org/10.1074/jbc.M112.415968

Publisher's Note Springer Nature remains neutral with regard to jurisdictional claims in published maps and institutional affiliations. 